\title{
Rechute extramédullaire d'une LAM 1 : présentation d'un cas avec une localisation gingivale
}

\author{
Boisramé-Gastrin $\mathbf{S}^{1}$, Tempescul $\mathbf{A}^{\mathbf{2}}$, Quintin-Roué $\mathbf{I}^{\mathbf{3}}$, Predine-Hug $\mathbf{F}^{\mathbf{1}}$ \\ ${ }^{1}$ UFR d'Odontologie, Service d'Odontologie, CHU, Brest, France \\ ${ }^{2}$ Service d'Hématologie, CHU, Brest, France \\ ${ }^{3}$ Laboratoire d'Anatomo-Pathologie, CHU, Brest, France \\ sylvie.boisrame-gastrin@chu-brest.fr
}

La réussite du traitement par allogreffe de mœlle osseuse est corrélée à l'intensité du conditionnement chimiothérapique, à la protection du rejet de greffe ainsi qu'à l'effet antileucémique du greffon $(\mathrm{GVL})$. Une forme particulière de rechute est observée après allogreffe pour les patients atteints de leucémie.

Le cas rapporté est celui d'un homme de 54 ans. L'histoire de sa maladie s'articule autour d'une LAM 1 normocytaire, avec des anomalies du chromosome 7 et un réarrangement du gène MLL, diagnostiquée en mai 2006. Le patient a été traité selon le protocole LAM 2001 du GOELAMS. Il a ensuite reçu une thérapie cellulaire allogénique, géno-identique, avec un conditionnement nonmyéloablatif. Le traitement préventif de la GVHD a été rapidement arrêté en l'absence de GVHD. Sept mois plus tard, le patient a présenté une rechute sanguine et médullaire. Il a reçu une deuxième allogreffe géno-identique, à partir de son deuxième frère. Le conditionnement a été cette fois myéloablatif et aucune prévention de la GVHD n'a été effectuée. Seize mois après la deuxième allogreffe, le patient a présenté une rechute isolée intéressant le corps caverneux, traitée par radiothérapie. Une première injection de Mylotarg ${ }^{\circledR}$ - anticorps anti-CD33 - a été effectuée en consolidation. Quatre semaines après celle-ci, le patient a été adressé en urgence dans le service d'Odontologie, pour une tuméfaction gingivale. L'examen clinique exobuccal n'a retrouvé aucune adénopathie. L'examen endobuccal a montré une tuméfaction dure, en regard des prémolaires maxillaires gauches. L'examen radiologique n'a révélé aucune lésion. La réalisation d'un lambeau de pleine épaisseur a permis de se rendre compte que la tuméfaction était située dans l'épaisseur de la gencive. Une incision vestibulaire a mis en évidence une masse indurée d'un blanc laiteux. L'examen anatomo-pathologique a révélé une localisation gingivale de la LAM.

Les rechutes leucémiques post-allogreffe posent des questions délicates. L'injection des lymphocytes de donneurs permet parfois de les contrer (Schmidt et al. 2007). Pour les formes agressives, le traitement consiste à réaliser en général une deuxième allogreffe qui permet d'obtenir des rémissions complètes avec un chimérisme complet (Guillerm et al. 2008). Malheureusement on observe chez cette population des rechutes extramédullaires (REM) (Tempescul et al. 2008). Il existerait donc une population de cellules leucémiques ayant une affinité tissulaire extra-médullaire (Firas et al. 2008). Le traitement optimal dans les cas de REM après allogreffe reste incertain. L'apport de la gentuzumab ozogamicine, anticorps monoclonal recombinant humanisé, semble intéressant dans ces cas. Cependant, les données sont trop fragmentaires pour déterminer l'effet sur la survie des patients présentant une REM (Owonikoko et al. 2007). Bien que des rémissions prolongées aient été rapportées, le pronostic des REM, survenant après allogreffe dans les leucémies aiguës, reste sombre. 